\title{
Embedded Systems Security
}

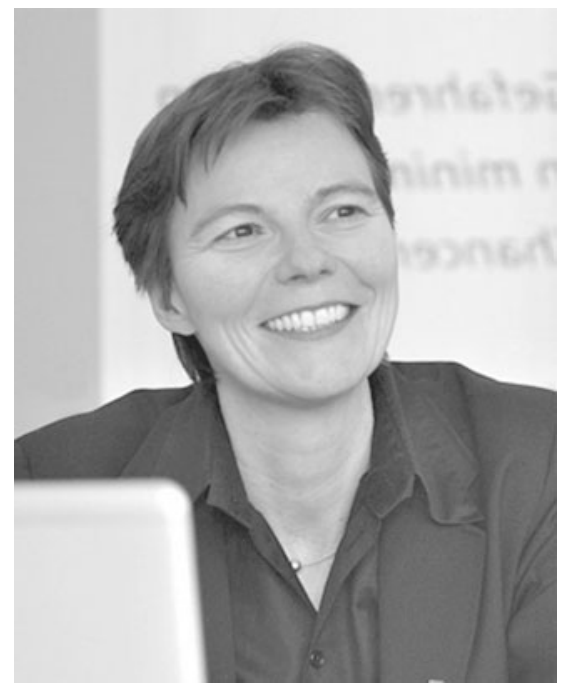

Die sichere Gestaltung und der verlässliche Betrieb komplexer, vernetzter, softwareintensiver Systeme zählen zu den größten Herausforderungen der IT-Sicherheit der kommenden Jahre. Eine besondere Bedeutung kommt hierbei der zunehmenden Integration von eingebetteten Systemen, als Herzstück derartiger Systeme, in physikalische Umgebungen und deren Verknüpfung mit betrieblichen Prozessen (Cyber-Physical Systems) zu. Sie sind bereits heute aus keiner der für unsere Gesellschaft wichtigen Anwendungsdomänen wie der Gesundheitsversorgung, der Produktion, der Logistik oder natürlich auch der Energieversorgung wegzudenken. Sie steuern und überwachen sicherheitskritische Abläufe und sind dazu auf vielfältige Weise miteinander vernetzt.

Dieses Themenheft beleuchtet ausgewählte Aspekte der Sicherheit eingebetteter Systeme. In Hardware-Komponenten implementierte, kryptographische Primitive bilden die Sicherheitsanker derartiger Systeme. Sie sind die vertrauenswürdigen Bausteine für Dienste zur Prüfung von Identitäten, zur vertraulichen Datenverarbeitung oder aber auch zur Erkennung von Manipulationen. Die Stärke kryptographischer Verfahren basiert auf der Güte der verwendeten Schlüssel und deren Geheimhaltung. Eingebettete Systeme sind in weit größerem Maß als Standard-PCs und Server anfällig gegen physikalische Attacken, da Angreifer häufig einen direkten physikalischen Zugriff auf sie haben. Man denke dabei nur an Smartcards, SmartMeter oder Sensoren im Fahrzeug. Die Palette der möglichen Angriffe reicht von einfachen Messungen, in Form von Seitenkanalangriffen, also passiven Verhaltensbeobachtungen, um Rückschlüsse auf die verwendeten kryptographische Verfahren zu ziehen, bis hin zu sehr komplexen Angriffen, die spezielle Geräte erfordern. Demensprechend sind Hardware-Bausteine zu entwickeln, die neben einem ausreichenden Sicherheitsniveau auch Optimierungsziele, wie einen geringen Stromverbrauch, geringen Flächenbedarf oder auch eine hohe Ausführungsgeschwindigkeit erfüllen. Zwei Beiträge widmen sich dieser Thematik. Stefan Mangard von Infineon gibt einen Überblick über physikalische Angriffe auf eingebettete Systeme und erläutert dann geeignete Schutzmaßnahmen zur Abwehr dieser Attacken. Ergänzend dazu konzentriert sich der Beitrag von Ingrid Verbauwhede von der KU Leuven auf die Frage der Entwicklung möglichst leichtgewichtiger und effizienter Kryptographie-Bausteine, die unterschiedliche Sicherheitslevel erfüllen, so dass damit eine Palette von Anwendungsgebieten, die sichere eingebettete Komponenten benötigen, abgedeckt wird.

Neben der Fragestellung der effizienten vertraulichen Kommunikation mit sicheren Schlüsselspeichern und einer sicheren Schlüsselgenerierung ist für eingebettete Systeme auch die Frage der Manipulations- und Fälschungssicherheit von großer Bedeutung. Zudem erfordert die Zunahme an autonomer Machine-to-Machine Kommunikation, dass sich eingebettete Komponenten verlässlich und effizient wechselseitig identifizieren können, also eine unverfälschbare Identität besitzen. Lösungen für diese Herausforderungen könnten neue physikalische Strukturen liefern, die bereits seit mehreren Jahren intensiv in der Forschung untersucht werden. Hierzu gehören Physical Unclonable Functions (PUF). Hierbei handelt es sich um physikalische Strukturen, die einzigartige physikalische Eigenschaften aufweisen. Der Artikel von Dominik Merli und Georg Sigl von der TU München und dem Fraunhofer AISEC gibt einen Überblick über Ansätze zur Umsetzung derartiger Strukturen in CMOS Schaltkreisen, wie sie in herkömmlichen Chipsätzen üblicherweise verwendet werden. Merli und Sigl erläutern Ansätze zur Nutzung der Strukturen zur Ableitung von unverfälschbaren Identitäten, zur Nutzung der Strukturen als sichere Schlüsselspeicher sowie zur Erkennung von Manipulationen. PUFs sind zusätzliche Schutzkonzepte, die auf einen Chip aufgebracht werden müssen. Damit bieten sie aber auch gleichzeitig neue Angriffsflächen. Hier setzt der Beitrag von Stefan Katzenbeisser und André Schaller von derTU Darmstadt an. Die Autoren beschreiben Angriffe auf PUF-Strukturen, skizzieren Abwehrmaßnahmen und erläutern eine Bandbreite möglicher Anwendungsfelder für PUFs.

Der Beitrag von Benedikt Driessen und Christof Paar von der Ruhr-Universität Bochum widmet sich dem dedizierten Anwendungsbereich der Sattelitentelefonie nach den ETSI-Standards GMR-1 und GMR-2. An Hand eines Angriffs auf die Satellitentelefonie zeigen die Autoren die Unsicherheit der GSMR-basierten Kommunikation. Ein Abhören der Kommunikation ist auch aus großer Distanz möglich, so dass die Privatsphäre der Kommunikationspartner in sehr viel größerem Maß gefährdet ist als bei herkömmlicher Mobiltelefonie mittels GSM.

Die Beiträge zum Schwerpunktthema Embedded System Security verdeutlichen die Vielfalt und Bandbreite der Fragestellungen im Bereich. Um neue Entwicklungen auch im Markt umzusetzen, ist eine sehr enge Zusammenarbeit zwischen Forschung, Hersteller-Unternehmen und Anwendungsbranche, unerlässlich.

Claudia Eckert 